\title{
Rezension zu: Follert, Florian, Zur Unternehmensbewertung im Spruchverfahren aus interessentheoretischer Sicht
}

\author{
Der aktienrechtliche Minderheitenausschluss im Lichte der Neuen \\ Politischen Ökonomie. Springer Gabler, Wiesbaden 2020, ISBN \\ 978-3-658-28922-5, €64,99
}

\section{Wolfgang Ballwieser}

Online publiziert: 28. Februar 2020

(C) Der/die Autor(en) 2020

Ziel der an der Universität des Saarlandes angenommenen Dissertation ist es ,einerseits, die Verbreitung der finanzierungstheoretischen Unternehmensbewertung in der Rechtsprechung der Spruchgerichte empirisch zu überprüfen, diese der Prüfung durch den Normzweck zu unterziehen und andererseits ein Erklärungsmodell für beobachtete Entwicklungen herauszuarbeiten“ (S. 9). In fünf Kapiteln beschäftigt sich der Verfasser hierzu mit der Unternehmensbewertung im Einflussbereich der Jurisprudenz (S. 1-10), als Gegenstand des Gesellschaftsrechts (S. 11-96), in Wissenschaft und rechtlicher Anwendung (S. 97-155) und der interessengeleiteten Rechtsprechung (S. 157-224), bevor er zusammenfasst und Ausblick bietet (S. 225-233). Literaturverzeichnis (S. 235-289) und zwei Anlagen (S. 291-361) beschließen die Arbeit.

Seine Ergebnisse erzielt der Verfasser durch Bezug auf die sog. Kölner Funktionenlehre und die insbesondere auf Manfred J. Matschke, Gerrit Brösel und Thomas Hering basierende Gegenüberstellung von investitions- und finanzierungstheoretischem Bewertungsansatz (S. 20). Der erste diene der Grenzpreisermittlung. Der zweite diene der Ermittlung eines hypothetisch objektiven Marktgleichgewichtspreises. Die Rechengrößen seien einerseits der dem Bewertungssubjekt zufließende Nettozahlungsstrom, andererseits diverse Cashflows. Das Hauptziel des ersten Ansatzes sei Entscheidungsunterstützung bei Lösung realer Bewertungsprobleme, das des zweiten Ansatzes Erklärung von Marktprozessen und Marktergebnissen.

Der Verfasser konzentriert sich zwar auf die Ermittlung der angemessenen Abfindung bei aktienrechtlichen Squeeze-outs, betrachtet diesen Anlass aber stellvertretend für andere gesellschaftsrechtliche Abfindungsfälle. Er sieht den Normzweck der Abfindungsermittlung in der - bei üblicherweise entstehenden Streitfällen - rich-

W. Ballwieser (ه)

LMU München, München, Deutschland

E-Mail:wball@bwl.lmu.de 
terlichen Ermittlung eines Schiedswerts auf Basis des subjektiven Grenzpreises von Abfindendem (Mehrheitsgesellschafter) und subjektivem Grenzpreis des ,relevanten Grenzminderheitsaktionärs“ (S. 92). Letzterer ist derjenige real existierende Aktionär mit dem niedrigsten Kalkulationszinsfuß (S. 91). Bei vorliegendem Einigungsbereich, d.h. Grenzpreis der Mehrheit größer Grenzpreis der Minderheit, müsse der Richter einen Wert aus diesem Bereich wählen, bei dessen Fehlen wäre - wegen Vorliegens einer dominierten Konfliktsituation - der Grenzpreis des derart typisierten Minderheitsgesellschafters relevant. Das Konzept findet sich insbesondere bei Matschke/Brösel (Unternehmensbewertung, 4. Aufl. 2013, S. 593). Die jeweiligen Grenzpreise seien nach dem investitionstheoretischen Bewertungsansatz mittels der Schätzung von Zukunftserfolgswerten zu ermitteln, wobei unsicherheitsaufdeckende statt unsicherheitsverdichtende Ansätze zu verwenden wären, was insbesondere Hering vertritt (Unternehmensbewertung, 3. Aufl. 2014, S. 182-200).

Diese Vorgehensweise findet sich aber - wie der Verfasser zeigt - weder (oder lediglich kaum) in den wissenschaftlichen betriebswirtschaftlichen Zeitschriften noch in bekannten aktienrechtlichen Kommentaren oder in der Rechtsprechung, weil dort - wie insbesondere in den Verlautbarungen des Instituts der Wirtschaftsprüfer - der finanzierungstheoretische Bewertungsansatz, der nur zur Argumentationswertermittlung tauge, vorherrscht. Deshalb verlässt der Verfasser im Hauptteil seiner Arbeit das Spielfeld der materiellen Unternehmensbewertung, um daran anschließend eine prozessuale Sicht einzunehmen. „Ziel ist es, die Wechselwirkungen der an der rechtlichen Unternehmensbewertung beteiligten Akteure zu untersuchen. Es soll herausgearbeitet werden, welche Spieler die Unternehmensbewertung in der Rechtsprechung prägen und beeinflussen.“ (S. 158) Hierzu wird auf einer Arbeit von Tobias Quill (Interessengeleitete Unternehmensbewertung 2016) aufgebaut. Danach zeigt sich, dass Minderheitsaktionäre vorrangig an einer zweckadäquaten Bewertung orientiert sind, während Hauptaktionär, Sachverständige i. S. v. WP und Richter als Interessenschnittmengen zum einen Glaubwürdigkeit, zum anderen Praktikabilität präferieren (S. 190). Daraufhin werden Mittel und Ergebnisse der Interessendurchsetzung behandelt (S. 191-224). Der Verfasser gelangt zum Resultat, dass der Minderheitsaktionär nach heutigem Stand im Zusammenspiel der genannten drei Interessengruppen den Kürzeren zieht und entfaltet Verbesserungsvorschläge zur Stärkung seiner Interessendurchsetzung.

Die Arbeit hat ein klares Ziel und eine nachvollziehbare Struktur und der Verfasser beweist Fleiß und Angriffslust, gepaart mit klarer Sprache. Daraus lässt sich freilich nicht folgern, dass er mich auch sachlich überzeugt hat. Ich möchte dies an einigen Punkten verdeutlichen:

Beim investitionstheoretischen Bewertungsansatz wird ein Überblick über Totalund Partialmodelle gegeben und eine Präferenz für das Zukunftserfolgswertverfahren geäußert. „Aus Gründen der Komplexitätsreduktion wird (...) nicht das gesamte Entscheidungsfeld des Bewertungssubjekts betrachtet, sondern das Bewertungsobjekt im Vergleich zur vermuteten besten Alternativverwendung bewertet" (S. 41) Als besonders fruchtbar erweise sich die Kombination dieses Verfahrens mit unsicherheitsaufdeckenden Methoden (wie einer Sensitivitäts- oder Risikoanalyse) betrachtet (S. 42). Für eine Kombination mit der Risikoanalyse wird auf Abschn. 2.3.2.3. (S. 88-95) verwiesen (S. 42, Fn. 201). Hier findet sich zwar ein Beispiel (S. 92-95), 
das der Verfasser selbst als „fiktives Fallbeispiel“ (S. 92) charakterisiert. Auf die Anwendung auf einen realistischeren Fall und dabei eventuell zu lösende Probleme geht der Verfasser jedoch weder hier noch anderswo ein. Es würde mich interessieren, wie er sich vorstellt, dass ein Sachverständiger für Abfindenden und Abzufindenden das Verfahren dem Richter so unterbreiten kann, dass dieser den Normzweck angemessen erfüllen kann. Hier schwebt der Verfasser in einsamer Höhe.

DCF-Verfahren, objektiviertes Ertragswertverfahren nach IDW S1, Multiplikator- und Sachoptionsverfahren werden der Überschrift ,Die Ermittlung von Argumentationsverfahren" subsumiert (S. 44-58). Wer bei DCF-Verfahren wegen ihres Gegenwartskalküls das bewertungstheoretische Fundament in der Investitionstheorie sehe, „offenbart indes mangelndes bewertungstheoretisches Hintergrundwissen. Diese Differenzierung ist essentiell, denn insbesondere, weil sie nicht auf der Investitionstheorie, sondern der Finanzierungstheorie fußen, eignen sich die, discounted cash flow'-Ansätze nicht zur Ermittlung von Entscheidungswerten." (S. 44) Es handele sich um „ein vom Individuum abstrahierendes Bewertungskonzept“ (S. 45) und bei Stützung des Zinssatzes auf das CAPM um einen objekt- statt subjektspezifischen Zinssatz. Mit Verweis auf Coenenberg/Schultze (FS Sieben, 1998) gelte: „Bewertungsrelevant sind hierbei nicht die Ausschüttungen, die zwischen dem Bewertungsobjekt und dem Bewertungssubjekt fließen, sondern ,cash flows' auf Unternehmensebene.“ (S. 46) Bei der „flow to equity“-Methode sei zwar „hinsichtlich der grundsätzlichen Methodik im Sinne eines Barwertkalküls (...) eine Ähnlichkeit mit dem Zukunftserfolgswertverfahren erkennbar. In Abgrenzung hierzu werden jedoch (...) sogenannte - meist uneinheitlich definierte - ,freie cash flows' mit einem Kapitalisierungszinssatz, der aus kapitalmarkttheoretischen Gleichgewichtsmodellen abgeleitet wird, diskontiert" (S. 46).

Beide Argumente sind oberflächlich, weil zum einen umfangreiche Bücher (z.B. Drukarczyk/Schüler, Unternehmensbewertung, 7. Aufl. 2016) zeigen, wie auf Unternehmensebene bezogene Cashflows mit Ausschüttungen korrespondieren und dass eine kompetenzbehaftete Anwendung verschiedener DCF-Verfahren zu ein und demselben Unternehmenswert führt. Zum anderen stimmt zwar der Verweis, dass eine sich am CAPM orientierende risikoangepasste Renditeforderung auf einem Gleichgewichtsmodell basiert. Das macht es aber für eine praktische Anwendung nicht völlig ungeeignet. Erstens könnte es die Bestimmung erwarteter oder realisierter Renditen am Aktienmarkt erklären. Bei schlechter Erklärung könnte es ergänzt oder aufgegeben werden. Weniger Kritik auf sich ziehende Ergänzungen (wie Mehr-Faktoren-Modelle oder Implizite Kapitalkosten) liegen jedoch auch nicht vor. Also ließe sich nur (aber auch: immerhin!) aus der Not eine Tugend machen. Zweitens gibt der Verfasser beim investitionstheoretischen Ansatz selbst ,zu bedenken, dass die periodenspezifischen Grenzzinsfüße auf einem unvollkommenen Kapitalmarkt ex ante oftmals nicht bekannt sind“" (S. 41). Was macht nun damit der Bewerter? Im Beispiel des Verfassers zur Verbindung von Zukunftserfolgswertverfahren und Risikoanalyse lesen wir auf S. 93: „Der typisierte endogene Grenzzinssatz des Minderheitsaktionärs wird durch den Sachverständigen auf 4\% geschätzt.“ Es wäre interessant zu erfahren, wie der Sachverständige eine solche Schätzung vor Gericht plausibel machen kann. Damit wird keineswegs behauptet, dass eine CAPM-Grundlage eine objektive Bestimmung des Zinsfußes ermögliche. Jeder Bewerter hat hierbei eine Vielzahl von 
Entscheidungen zu fällen, die in der Literatur hinreichend dokumentiert sind. Er tut gut daran, sie zu explizieren. Das gelingt ihm aber vermutlich leichter, als wenn er über endogene Grenzzinssätze eines relevanten Grenzminderheitsaktionärs (s. oben) reflektiert.

Im Zusammenhang mit der Entstehung von IDW S1 und dessen Öffnung für finanzierungstheoretische Konzepte wird meine Rolle im Arbeitskreis Unternehmensbewertung (AKU) des IDW ,äußerst kritisch“ beurteilt (S. 52), nicht zuletzt, weil ich doch vielfach auf die Schwächen des CAPM hingewiesen hätte. Meine Funktion des Wissenschaftlers und meine Funktion des AKU-Mitglieds seien unvereinbar gewesen. Meine Rolle im AKU wird damit überschätzt, die „,reine Lehre“ des investitionstheoretischen Ansatzes wird damit missionarisch gepredigt und mein denkbares Ringen um noch vertretbare Lösungen wird erst gar nicht thematisiert. Freilich frage ich mich, ob das im Zusammenhang mit dem Thema der Arbeit überhaupt von Interesse ist.

Die Kritik am IDW ist vielfältig und heftig. Hierbei lässt sich vieles nachvollziehen. Dennoch ist zu fragen, wie sich der Verfasser die Unterstützung der Richter bei Spruchverfahren durch Sachverständige vorstellt. Sind das dann allein unabhängige Hochschullehrer? Der Verfasser hat diesbezüglich gewisse Zweifel (S. 232). Oder werden die Wirtschaftsprüfer im Sinne des investitionstheoretischen Bewertungsansatzes neu geschult, obwohl dieser Ansatz doch erkennbare Akzeptanzgrenzen in der Wissenschaftsgesellschaft erfährt? Sein Ergebnis lautet: „Es kann den Interessenvertretungen des Minderheitsaktionärs empfohlen werden, auf die Unterstützung solcher Wissenschaftler zurückzugreifen, die sich kritisch mit der finanzierungstheoretischen Unternehmensbewertung im Rahmen der Rechtsprechung auseinandersetzen. Hier sollte ein Gegenpol zu der ebenfalls auf die wissenschaftliche Expertise bauenden Interessenvertretungen des Sachverständigen und des Hauptaktionärs entworfen werden. Im Rahmen der Facharbeit sollte deutlich artikuliert werden, dass lediglich eine funktionale Unternehmensbewertung, welche auf einem investitionstheoretischen Fundament basiert, in der Lage ist, die Interessen des Minderheitsaktionärs zu schützen und damit dem Normzweck zu entsprechen." (S. 232).

Zusammenfassend gilt: Die Arbeit widmet sich einem spannenden und keineswegs ausdiskutierten Thema. Sie ist mit nahezu missionarischem Eifer geschrieben, hat ein klares Feindbild und eindeutige Kriterien für Gut und Böse. Grautöne oder Maß und Mitte drohen ihr abzugehen. Da radikale Sichtweisen für die Fortentwicklung von Theorien oder die Einschätzung realer Gegebenheiten per se kein Nachteil sind, wünsche ich ihr viele Leser. Meinen Eindruck habe ich geschildert. Wenn er falsch sein sollte, können Leser von Werk und Rezension leicht zu anderen Ergebnissen gelangen.

Funding Open Access funding provided by Projekt DEAL.

Open Access Dieser Artikel wird unter der Creative Commons Namensnennung 4.0 International Lizenz veröffentlicht, welche die Nutzung, Vervielfältigung, Bearbeitung, Verbreitung und Wiedergabe in jeglichem Medium und Format erlaubt, sofern Sie den/die ursprünglichen Autor(en) und die Quelle ordnungsgemäß nennen, einen Link zur Creative Commons Lizenz beifügen und angeben, ob Änderungen vorgenommen wurden. 
Die in diesem Artikel enthaltenen Bilder und sonstiges Drittmaterial unterliegen ebenfalls der genannten Creative Commons Lizenz, sofern sich aus der Abbildungslegende nichts anderes ergibt. Sofern das betreffende Material nicht unter der genannten Creative Commons Lizenz steht und die betreffende Handlung nicht nach gesetzlichen Vorschriften erlaubt ist, ist für die oben aufgeführten Weiterverwendungen des Materials die Einwilligung des jeweiligen Rechteinhabers einzuholen.

Weitere Details zur Lizenz entnehmen Sie bitte der Lizenzinformation auf http://creativecommons.org/ licenses/by/4.0/deed.de. 